\title{
Primary Vaginal Melanoma, A Rare and Aggressive Entity. A Case Report and Review of the Literature
}

\author{
EMMANOUIL KALAMPOKAS ${ }^{1}$, THEODOROS KALAMPOKAS ${ }^{2}$ and CHRISTOS DAMASKOS ${ }^{3,4}$ \\ ${ }^{1}$ Department of Gynaecological Oncology, Aberdeen Royal Infirmary, Aberdeen, U.K; \\ ${ }^{2}$ Second Department of Obstetrics and Gynecology, Aretaieion Hospital, \\ University of Athens Medical School, Athens, Greece; \\ ${ }^{3}$ Second Department of Propedeutic Surgery, Laiko General Hospital, \\ National and Kapodistrian University of Athens, Medical School, Athens, Greece; \\ ${ }^{4}$ N.S. Christeas Laboratory of Experimental Surgery and Surgical Research, \\ Medical School, National and Kapodistrian University of Athens, Athens, Greece
}

\begin{abstract}
Malignant melanoma of the vagina is a rare, aggressive malignancy of poor prognosis. It principally affects post-menopausal women, with a mean age of 57 years, and the factors that contribute to its appearance are not well known. The first case of primary malignant vaginal melanoma was reported in 1887 and modern literature has noted about 500 cases, globally. Vaginal melanomas constitute $0.3 \%$ of all malignant melanomas and fewer than $3 \%$ of all vaginal carcinomas. To date there is no clear consensus regarding treatment. An early, accurate diagnosis and prompt investigation is essential in reaching appropriate treatment decisions. We present a clinical case of primary vaginal melanoma and review the literature briefly, presenting the current treatment plans and updates of this rare gynecological malignancy. Considerations, epidemiology, associated risk factors, response to therapy and expected outcome are also discussed. Conclusion: Primary malignant vaginal melanoma is a rare but aggressive melanoma that affects women in their 6th and 7th decade of life. The tumor appears as a dark node or spindle but can also be amelanotic. The size of the tumor is indicative of the prognostic factors. Surgery seems to be the only efficient treatment. Postoperative adjuvant therapy might help in preventing recurrence of the tumor. The survival rate is largely dependent on nodal and distant metastasis of the disease after initial tumor resection. There is a dire need to form a proper therapeutic regime to control this disease.
\end{abstract}

This article is freely accessible online.

Correspondence to: Christos Damaskos, MD, M.Sc., Ph.D., 17 Agiou Thoma Street, Athens, 11527, Greece. Tel: +30 6948467790, Fax: +30 2132061772, e-mail: x_damaskos@yahoo.gr

Key Words: Primary, vaginal, melanoma, excision, staging, treatment, prognosis.
Melanomas are tumors that arise from melanocytes or the pigment cells. A common form of melanoma is primary mucosal melanoma that is found in the mucosal lining of the urogenital tract, respiratory tract and the gastrointestinal tract (1). In $3 \%$ of healthy women, melanocytes can be found in the basal portion of the vaginal epidermis, as a embryological remnants of neural crest cells (2). Primary malignant vaginal melanoma is one such type of mucosal malignant melanoma that is very rare (3) and very aggressive in nature (4). Multifocal development of non-cutaneous melanomas at a location that is not exposed to ultraviolet rays with an incidence less than that of cutaneous melanomas has been reported (5). Primary malignant vaginal melanoma is thought to arise from aberrantly located melanocytes of vaginal epidermis (2).

The first case of primary malignant vaginal melanoma was reported in 1887 and modern literature has noted about 500 cases, globally. This constitutes $0.3 \%$ to $0.8 \%$ of melanomas in women, fewer than $3 \%$ of vaginal malignant tumors and fewer than $10 \%$ of female genital tract melanomas (6). Even though the age interval of the disease has been stated to be $38-90$ years, it is often observed in elderly women in their 6 th or 7 th decade of life (5), where lymph node infiltration and distant metastasis reach $50 \%$ and $20 \%$ respectively (7), owing to the ability of tumor to spread hematogenously and regardless of primary therapy chosen (2).

The disease presents poor prognosis even in the case of localized lesions (4), whereas lymph node and distant metastasis have been linked to reduced chance of survival. Although there is no effective treatment strategy yet in place (8), the current treatment plan includes wide local excision (WLE), radical surgery, chemotherapy, immunotherapy, combination therapeutics and palliative care (3). Radiotherapy is used as adjuvant therapy, as data suggest that radiotherapy does not bring overall benefit as sole 
therapy (9). Evidence from case reports suggests that the overall 5-year survival rate of patients with primary malignant vaginal melanoma is $0-25 \%$, irrespective of the type of treatment followed (7).

\section{Case Report}

We present a clinical case of primary malignant vaginal melanoma in a 75 years of age, para 2, Caucasian woman referred with post-menopausal bleeding. Her smear history was normal and she did not have any history of malignancy among first-degree relatives.

On gynaecological examination, a $5 \mathrm{~mm}$ raised, hyperpigmented lesion was seen on the anterior vaginal wall, which bled easily on contact. There were no palpable inguinal lymph nodes. A punch biopsy was taken and the pathology report came back as primary malignant vaginal melanoma with a maximum tumor thickness of $4.85 \mathrm{~mm}$, a high mitotic figure rate, and positive immunohistochemical staining for vimentin, MART-1 and AE1/AE3. Preoperative evaluation of the patient with computerized tomography (CT) of the chest, abdomen and pelvis, and magnetic resonance imaging of the brain did not demonstrate signs of local spread, lymphadenopathy or distant metastases.

Since there is no clear benefit in overall survival after radical surgery, the patient chose to undergo WLE of the tumor with at least $1.5 \mathrm{~cm}$ free margins (Figure 1). The postoperative histological diagnosis was confirmed by positive immunohistochemical staining for $\mathrm{S}-100$ and MART-1 as primary malignant vaginal melanoma with clear surgical margins. In addition, the tumor cells did not demonstrate BRAF or KIT mutations (Figure 2). The inguinal nodes were not removed since there were no signs of lymphadenopathy preoperatively. The patient refused any additional treatment such as radiotherapy or chemotherapy. Twelve months after the initial diagnosis and follow-up at 3month intervals with clinical examination and CT of chest, abdomen and pelvis there remain no signs of disease recurrence.

\section{Discussion}

Epidemiology and associated risk factors. According to a review by Leitao et al., a period of 29 years registered only 37 cases of malignant vaginal melanoma. Similarly, in a study by the Gynecologic Oncology Group spanning 17 years recorded only 43 such cases (10). The annual estimated incidence of primary malignant vaginal melanoma is 0.026 per 100,000 women (7) and 0.46 per one million women (8). A statistical analysis by Shah et al. on the epidemiology of malignant vaginal cancer indicated that among the 2.531 cases taken under study, non-Hispanic Whites constituted $66 \%$ of the registered cases (11); African American women,
Hispanic women, Asian/Pacific Islanders and others constituted $14 \%, 12 \%, 7 \%$ and $1 \%$ of the total number of malignant vaginal cancer cases. Hu et al. suggested that the low difference in the incidence rates of various racial and ethnic groups indicates that race is not a risk factor for primary malignant vaginal melanoma (12).

The incidence rates have been found to be very high for post-menopausal women, ranging between 2.08/100,000 for 60 - to 69 -year-old women and $4.43 / 100,000$ for 80 - to 89 year-old women (11). The average age at first diagnosis was found to be 57 years (7). Socioeconomic factors such as level of education, income and poverty seem to have no influence on incidence rates (11).

Etiology and pathogenesis. Currently, the mechanism of pathogenesis and etiological factors for primary malignant vaginal melanoma are unknown. This mystery extends to other types of melanomas. Whiteman et al. proposed a series of theories to elucidate the possible causes of melanomas. The difference in the incidence rate of occurrence of melanoma across sun-exposed and unexposed sites on the body suggest that the anatomic location of the melanocytes determines the mechanisms by which they interact with UV radiation (13). The theories suggested that even though UV radiation does not have a strong causal relationship with melanoma, the marginally increased prevalence of the disease in Caucasians acts as compelling evidence to reconsider this suggestion (12).

The theories also propose that melanomas with $B R A F$ mutations could be spread out at multiple locations in the body, implicating this gene in triggering the formation of nevi (skin abnormalities), although not enough to cause melanoma. However, primary malignant vaginal melanoma is a mucosal melanoma and mucosal melanomas are more often associated with $K I T$ gene mutations rather than $B R A F$ mutations, which are more often found in cutaneous melanomas (13). KIT encodes for a protein called c-KIT. In two different studies on mucosal melanoma, $39 \%$ and $15.6 \%$ of cases exhibited multiple copies of $\operatorname{KIT}(14,15)$. Another theory suggests that the causative factor could be microenvironmental in nature and not associated with ultraviolet radiation (12).

Clinical symptoms and signs. Nodularity describes the majority of the lesions (5). A tumor is designated as primary malignant vaginal melanoma when the primary site of the tumor is the vagina, which commences from the vulva and ends upward at the uterine cervix (16). Primary malignant vaginal melanoma usually occurs in the anterior wall of the vagina, typically in the lower one-third, as in our case (Figure 1) $(8,17)$. However, there are several case reports suggesting that the tumor can also arise in the upper third (4, 5,18 ). In a study by Xia et al., $54.6 \%$ of patients had tumors 


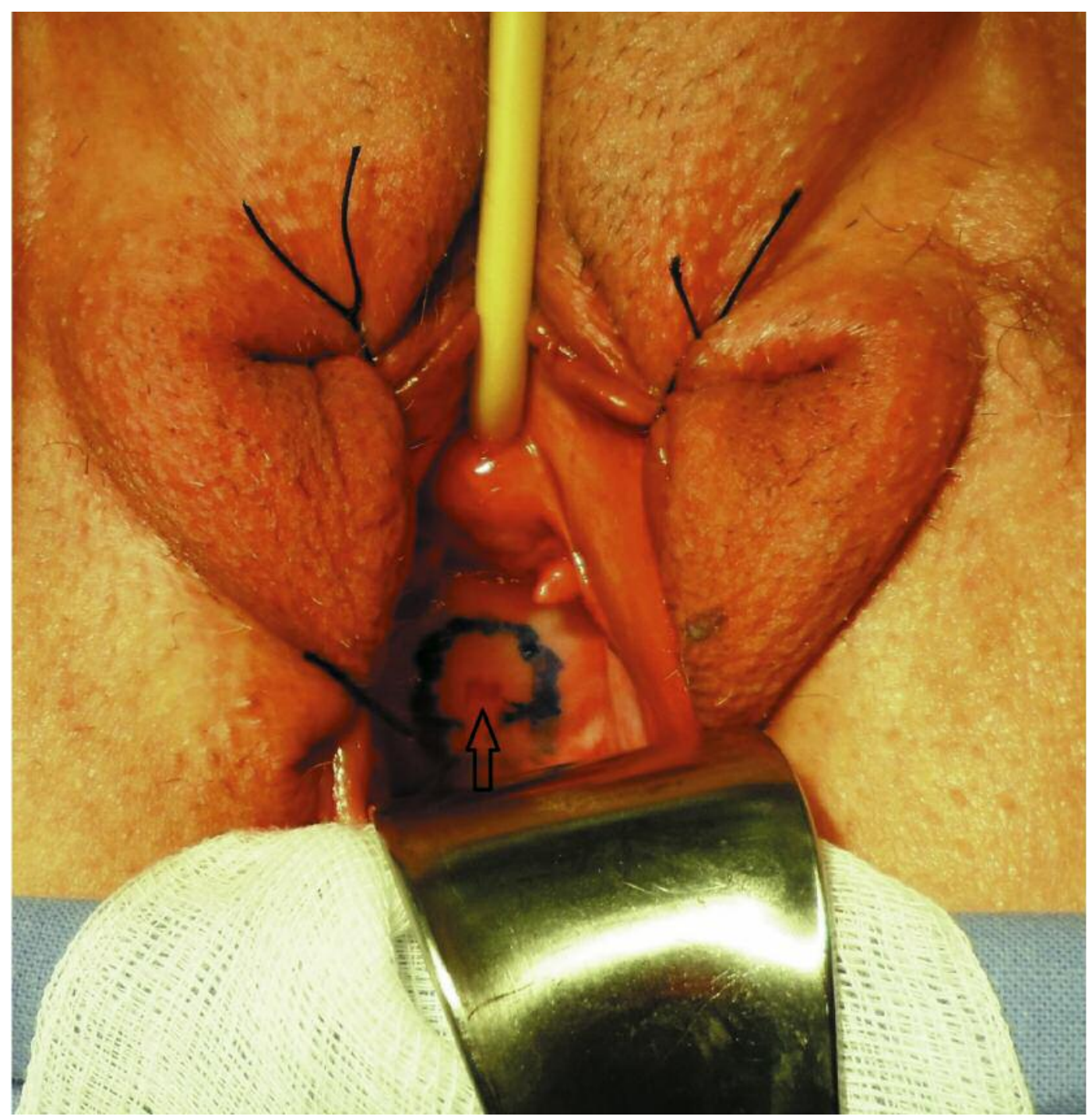

Figure 1. A lesion (black arrow) of primary vaginal melanoma on the anterior vaginal wall.

in the distal one-third of the vagina (19). Gökaslan et al. reported malignant vaginal melanoma cases presenting lesions on the posterior wall near the vaginal entrance (2), while Shah et al. reported the presence of pigmented tumors in the middle and lower thirds of the vagina (11).

Common symptoms of primary malignant vaginal melanoma are abnormal vaginal bleeding (63.6-80\%), presence of a vaginal mass that is easily palpable (15$15.9 \%)$, pain $(2.3-10 \%)$ and discharge from the vagina (15.9$25 \%)(4,19)$.

Diagnosis. Upon visual inspection, the tumor may appear nodular (pigmented mass of brown, blue or black) or polypoid (polyp-like) in nature. The tumor usually bleeds on touch and is ulcerated in many patients (1). Although the tumor is often found as a pigmented mass, amelanotic tumors have also been recorded in patients, which often leads to misdiagnosis and delayed treatment $(1,4)$. The tumor is resected for biopsies (punch and not shaved) or its contents aspirated using a fine needle. The analysis includes an immunohistochemical analysis, visual examination and determination of the presence of mutations associated with melanomas (7). Histologically, the tumor cells are epithelioid in nature in $55 \%$ of cases, spindled in $17 \%$ and mixed type in $28 \%$ of cases (4). Primary melanoma is confirmed by positive immunohistochemical staining of protein S-100, melan A, human melanoma black 45 (HMB-45) and vimentin $(3,17,18)$. Differential diagnosis includes testing 

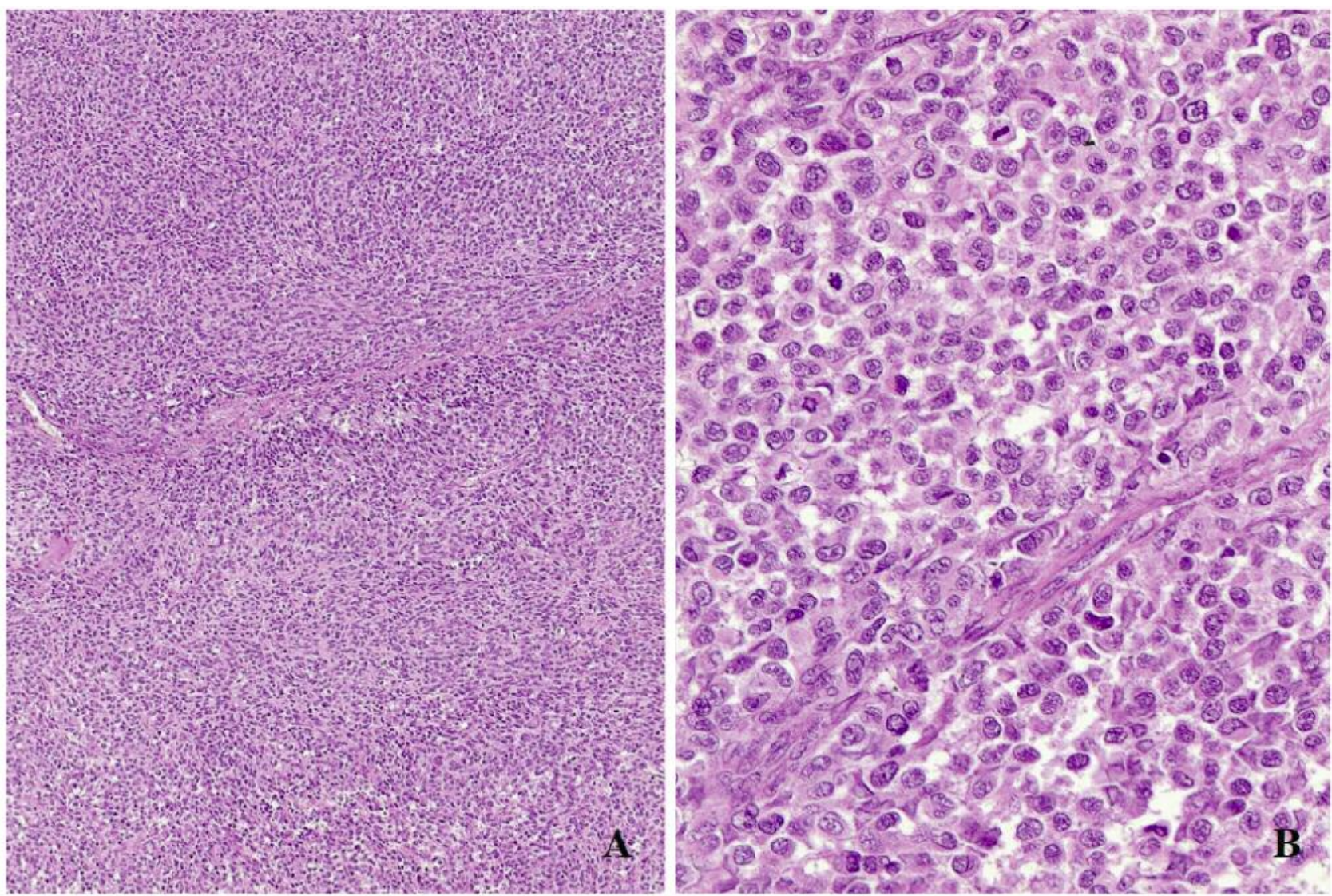

Figure 2. A. Malignant melanoma with a nodular growth pattern $(\times 100)$. B. High-power view $(\times 400)$ of neoplastic cells exhibiting large pleiomorphic nucleus and eosinophilic cytoplasm. Mitotic activity is prominent.

for cytokeratin, chromogranin, estrogen and progesterone receptors, that must be negative in order to confirm melanoma (20).

Pathological testing is carried out to determine the Breslow depth of the tumor (Table I) (21), mitotic number, ulceration, presence of microsatellites and the appearance of the margins (10). Imaging techniques such as computerized axial tomography (22), thoracic-abdominal-pelvic CT scan, positron-emission tomography - CT scan and X-ray are used for diagnosis (2). Testing for genetic mutation in KIT, BRAF and NRAS are performed during initial diagnosis (10). To test for metastasis, sentinel lymph node biopsy is performed (23).

Staging. Clinical staging of primary mucosal melanomas is based on guidelines by the International Federation of Gynecology and Obstetrics (FIGO), while pathological staging is based on the American Joint Committee on Cancer's (AJCC) TNM staging method (24). TNM has been typically used for staging skin cancer lesions (Table II) (6); however, due to its detailed staging system and prognosis prediction,
Table I. Staging by Breslow's depth.

\begin{tabular}{lc}
\hline Stage & Depth $(\mathrm{mm})$ \\
\hline I & $\leq 0.75$ \\
II & $0.76-1.50$ \\
III & $1.51-2.25$ \\
IV & $2.26-3.0$ \\
V & $\geq 3.0$ \\
\hline
\end{tabular}

Seifried et al. found it to be a better staging system for malignant vulva and vaginal melanomas (23), yet some literature reports use of both systems simultaneously (6).

According to FIGO, the tumors are staged from I to IVA and IVB depending on the location and infiltration of the tumor. Stage I malignant vaginal tumors are confined to the vaginal wall, while stage IVB have metastasized to distant organs (16). The TNM staging assesses the size of the 
Table II. International Federation of Gynecology and Obstetrics (FIGO) system of staging combined with the American Joint Committee on Cancer (AJCC) TNM system (24).

\begin{tabular}{lcc}
\hline TNM category & FIGO stage & \\
\hline Tumor (T) & & Primary tumor cannot be assessed \\
TX & & No evidence of primary tumor \\
T0 & Tumor confined to vagina \\
T1 & II & Tumor invades paravaginal tissues but not to pelvic wall \\
T2 & III & Tumor extends to pelvic wall \\
T3 & IVA & Tumor invades mucosa of the bladder or rectum and/or extends beyond the true pelvis \\
T4 & & Regional lymph nodes cannot be assessed \\
Regional lymph nodes (N) & No regional lymph node metastasis \\
NX & III & Pelvic or inguinal lymph node metastasis \\
N0 & & No distant metastasis \\
N1 & Distant metastasis \\
Distant metastasis (M) & IVB \\
M0 & \\
M1 & & Dis \\
\hline
\end{tabular}

primary tumor $(\mathrm{T})$, infiltration of the nearby lymph nodes $(\mathrm{N})$ and level of metastasis (M). Depending on the size of the tumor (no tumor to greater than $4 \mathrm{~mm}$ in thickness with ulceration), the tumor is classified from TX to T4b. Stages $\mathrm{T} 1$ to $\mathrm{T} 4$ are comparable to stages I to IVA of the FIGO system. Similarly, depending on metastasis to the lymph nodes, the tumor is classified from NX (no lymph node metastasis) to $\mathrm{N} 3$ (metastasis to four or more regional nodes). Distant metastasis staging is from MX (no metastasis) to M1c (metastasis to all visceral sites) (25).

Treatment. Surgery: For melanomas that can be resected, surgery is the preferred treatment (6). Moreover, surgical treatment has been found to be positively associated with prolonged survival when compared to other treatment methods (9). A popular surgical method for early stages of the tumor is the WLE method. WLE with a safety margin of $1 \mathrm{~cm}$ for tumors with a Breslow depth of $2 \mathrm{~mm}$ or less and $2 \mathrm{~cm}$ for tumors with a Breslow depth of more than $2 \mathrm{~mm}$ is performed along with pelvic radiotherapy $(2,10,19)$. In the series of Miner et al., the median survival of women with primary vaginal melanoma was calculated at 20 months. The patients who underwent surgery first were found to have a better survival compared to those in whom primary radiotherapy was performed. The thorough removal of the macroscopic tumor and negative free margins were found to be effective in guaranteeing local control and improving survival (26).

Radical surgery and lymphadenectomy with adjuvant chemo- or radiotherapy is used as an aggressive treatment for advanced stages of primary malignant vaginal melanoma $(2,17,22)$. However, lymphadenectomy is contraindicated in patients who show no lymph node metastasis $(2,10)$. Reports show that radical surgery can include total
Table III. Prognostic factors of primary vaginal melanoma.

\begin{tabular}{l}
\hline Prognostic factor \\
\hline Age \\
FIGO stage \\
Tumor location \\
Tumor size \\
Depth of invasion \\
Pigmentation \\
Ulceration \\
Histology \\
Cell type \\
Number of mitoses \\
Venous invasion \\
Type of surgical approach \\
Adjuvant radiotherapy \\
Chemotherapy
\end{tabular}

FIGO: International Federation of Gynecology and Obstetrics.

hysterectomy, vaginectomy and vulvectomy depending on the location of the tumor (proximal, middle or distal to the vagina) $(3,5,6,19)$.

Chemotherapy: In cases of inoperable tumor in advanced stages or deteriorating health condition of the patients, palliative chemotherapy is preferred, which does not cure the disease but helps prolong the life of the patients while managing pain. Chemotherapy is performed using dacarbazine (dimethyl-triazeno imidazole-carboxamide; DTIC), temozolomide (oral analog of DTIC) (4), or paclitaxel, nitrosourea, imatinib, nidran and vincristine (5). Paclitaxel, temozolomide and DTIC are sometimes used in combination with cisplatin with/without carboplatin (10). 
Biochemotherapy: In cases of advanced-stage disease, the combination of chemotherapy and immunotherapy (biochemotherapy) is associated with an increased response rate, but its effectiveness has not yet been established. When applied, it has been linked with increased toxicity $(27,28)$.

Postoperative adjuvant therapy. Immunotherapy: Postoperative adjuvant immunotherapy using interferon alpha- $2 b$ has been found to be effective in keeping the tumor from relapsing $(3,29)$. It is also one of the first agents that was approved by the US Food and Drug Administration for treatment of metastasized melanoma (10). Immunotherapy has also been performed using agents such as measles vaccine, dendritic cells, interleukin-2, lymphokine-activated killer cellsand bacillus Calmette-Guérin (29).

Radiotherapy: Radiotherapy has been found to be effective as a postoperative adjuvant therapy alongside chemotherapy and immunotherapy for tumors that cannot be removed surgically, but not as a sole treatment for primary malignant vaginal melanoma $(3,4,9)$. However, the literature shows that radiotherapy is also offered as a treatment regime if the patient refuses surgical intervention $(9,30)$. Moreover, it is applied preoperatively as adjuvant treatment for tumor shrinkage and achievement of a more conservative management, or postoperatively as adjuvant treatment in cases of incomplete tumor resection or with pelvic metastases (31).

Topical treatment: Topical treatment with $5 \%$ imiquimod was found to be effective as an adjuvant treatment in six patients by Smyth et al. (32) and Prescott et al. (33) with no evidence of recurrence for a period of 18 months.

Targeted therapy: Targeted therapy using a monoclonal antibody has been used for primary malignant vaginal melanoma prolonging survival and preventing recurrence of the tumor; however, no trials have been conducted specifically for malignant vaginal tumors (10).

Prognostic factors and survival. The potential prognostic factors include age, FIGO stage, tumor location, tumor size, depth of invasion, pigmentation, ulceration, histology, cell type, number of mitoses, venous invasion, type of surgical approach, adjuvant radiotherapy, and chemotherapy (5) (Table III).

Patients with tumors less than $3 \mathrm{~cm}$ in size have a better prognosis (survival of 41 months, approximately) when compared to those who present tumors larger than $3 \mathrm{~cm}$ (survival of 12 months, approximately) (1). The overall 5-year survival independent of treatment is reported to be $0-25 \%$ (26). A study by Kirschner et al. reported that the 5-year survival rate is $17 \%$ when the tumor is restricted to the vaginal wall and $15 \%$ for all other patients with primary malignant vaginal melanoma, even when no lymph nodes have been infiltrated. The survival rate with lymph node involvement dwindles to $5 \%$ within a 3 -year period. Patients who underwent surgical treatment have been shown to live longer when compared to those who received non-surgical treatments (9).

\section{Conclusion}

This review of current literature shows that primary malignant vaginal melanoma is a rare but aggressive melanoma that affects women in their 6th and 7th decade of life. The tumor appears as a dark node or spindle but can also be amelanotic. The size of the tumor is indicative of the prognostic factors. Surgery seems to be the only efficient treatment regime that significantly improves longevity of the patient. Postoperative adjuvant therapy using chemotherapy, radiation therapy, topical creams, immunotherapy and targeted therapy might help prevent recurrence of the tumor. The survival rate is largely dependent on nodal and distant metastasis of the disease after initial tumor resection. There is a dire need to form a proper therapeutic regime to control this disease.

\section{References}

1 Mihajlovic M, Vlajkovic S, Jovanovic P and Stefanovic V: Primary mucosal melanomas: a comprehensive review. Int $\mathrm{J}$ Clinical Pathol 5: 739-753, 2012.

2 Gokaslan H, Sismanoglu A, Pekin T, Kaya H and Ceyhan N: Primary malignant melanoma of the vagina: a case report and review of the current treatment options. Eur J Obstet Gynecol Reprod Biol 121: 243-248, 2005.

3 Lin LT, Liu CB, Chen SN, Chiang AJ, Liou WS and Yu KJ: Primary malignant melanoma of the vagina with repeated local recurrences and brain metastasis. J Chin Med Assoc 74: 376379, 2011.

4 Androutsopoulos G, Terzakis E, Ioannidou G, Tsamandas A and Decavalas G: Vaginal primary malignant melanoma: a rare and aggressive tumor. Case Rep Obstet Gynecol 2013: 137908, 2013.

5 Baloglu A, Bezircioglu I, Cetinkaya B and Yavuzcan A: Primary malignant melanoma of the vagina. Arch Gynecol Obstet 280: 819-228, 2009.

6 Schmidt M, Honig A, Schwab M, Adam P and Dietl J: Primary vaginal melanoma: a case report and literature review. Eur $\mathbf{J}$ Gynaecol Oncol 29: 285-288, 2008.

7 Kuhn F, Dieterich M, Klar E, Gerber B and Prinz C: Primary malignant vaginal melanoma - case report and review of the literature. Geburtshilfe Frauenheilkd 72: 740-743, 2012.

8 Chaudhuri S, Das D, Chowdhury S and Gupta AD: Primary malignant melanoma of the vagina: A case report and review of literature. South Asian J Cancer 2: 4, 2013.

9 Kirschner AN, Kidd EA, Dewees T and Perkins SM: Treatment approach and outcomes of vaginal melanoma. Int J Gynecol Cancer 23: 1484-1489, 2013.

10 Leitao MM Jr., Cheng $X$, Hamilton AL, Siddiqui NA, Jurgenliemk-Schulz I, Mahner S, Åvall-Lundqvist E, Kim K and Freyer G: Gynecologic Cancer InterGroup (GCIG) consensus review for vulvovaginal melanomas. Int J Gynecol Cancer 24(9 Suppl 3): S117-122, 2014.

11 Shah CA, Goff BA, Lowe K, Peters WA, 3rd and Li CI: Factors affecting risk of mortality in women with vaginal cancer. Obstet Gynecol 113: 1038-1045, 2009. 
$12 \mathrm{Hu}$ DN, Yu GP and McCormick SA: Population-based incidence of vulvar and vaginal melanoma in various races and ethnic groups with comparisons to other site-specific melanomas. Melanoma Res 20: 153-158, 2010.

13 Whiteman DC, Pavan WJ and Bastian BC: The melanomas: a synthesis of epidemiological, clinical, histopathological, genetic, and biological aspects, supporting distinct subtypes, causal pathways, and cells of origin. Pigment Cell Melanoma Res 24: 879-897, 2011.

14 Curtin JA, Busam K, Pinkel D and Bastian BC: Somatic activation of KIT in distinct subtypes of melanoma. J Clin Oncol 24: 4340-4346, 2006.

15 Beadling C, Jacobson-Dunlop E, Hodi FS, Le C, Warrick A, Patterson J, Town A, Harlow A, Cruz F 3rd, Azar S, Rubin BP, Muller S, West R, Heinrich MC and Corless CL: KIT gene mutations and copy number in melanoma subtypes. Clin Cancer Res 14: 6821-6828, 2008.

16 Hacker NF, Eifel PJ and van der Velden J: Cancer of the vagina. Int J Gynaecol Obstet 119 (Suppl 2): S97-99, 2012.

17 Samolis S, Panagopoulos P, Kanellopoulos N, Papastefanou I, Karadaglis S and Katsoulis M: Primary melanoma of the vagina: a case report. Eur J Gynaecol Oncology 31: 233-234, 2010.

18 Chen L, Xiong Y, Wang H, Liang L, Shang H and Yan X: Malignant melanoma of the vagina: A case report and review of the literature. Oncol Lett 8: 1585-1588, 2014.

19 Xia L, Han D, Yang W, Li J, Chuang L and Wu X: Primary malignant melanoma of the vagina: a retrospective clinicopathologic study of 44 cases. Int J Gynecol Cancer 24: 149-155, 2014.

20 Albareda J, Olier C and Alemany I: Primary melanoma of the vagina. A clinical case. J Turk Ger Gynecol Assoc 12: 50-52, 2011.

21 Breslow A: Thickness, cross-sectional areas and depth of invasion in the prognosis of cutaneous melanoma. Ann Surg 172: 902-908, 1970.

22 Moodley M, Daya M and Moodley J: Vaginal malignant melanoma: a case report and literature review. Int $\mathrm{J}$ Gynecol Cancer 14: 687-689, 2004

23 Seifried S, Haydu LE, Quinn MJ, Scolyer RA, Stretch JR and Thompson JF: Melanoma of the vulva and vagina: principles of staging and their relevance to management based on a clinicopathologic analysis of 85 cases. Ann Surg Oncol 22: 1959-1966, 2015.
24 Edge SB and Compton CC: The American Joint Committee on Cancer: the 7th edition of the AJCC cancer staging manual and the future of TNM. Ann Surg Oncol 17: 1471-1474, 2010.

25 Greene FL: The American Joint Committee on Cancer: updating the strategies in cancer staging. Bull Am Coll Surg 87: 13-15, 2002.

26 Miner TJ, Delgado R, Zeisler J, Busam K, Alektiar K, Barakat $\mathrm{R}$ and Poynor E: Primary vaginal melanoma: a critical analysis of therapy. Ann Surg Oncol 11: 34-39, 2004.

27 Ives NJ, Stowe RL, Lorigan P and Wheatley K: Chemotherapy compared with biochemotherapy for the treatment of metastatic melanoma: a meta-analysis of 18 trials involving 2,621 patients. J Clin Oncology 25: 5426-5434, 2007.

28 Harting MS and Kim KB: Biochemotherapy in patients with advanced vulvovaginal mucosal melanoma. Melanoma Res 14: 517-520, 2004.

29 Huang Q, Huang H, Wan T, Deng T and Liu J: Clinical outcome of 31 patients with primary malignant melanoma of the vagina. J Gynecol Oncol 24: 330-335, 2013.

$30 \mathrm{Ng}$ YK, Lee JY, Supko KM, Khan A, Torres SM, Berwick M, Ho J, Kirkwood JM, Siegfried JM and Stabile LP: Pan-erbB inhibition potentiates BRAF inhibitors for melanoma treatment. Melanoma Res 24: 207-218, 2014.

31 Piura B: Management of primary melanoma of the female urogenital tract. Lancet Oncol 9: 973-981, 2008.

32 Smyth EC, Flavin M, Pulitzer MP, Gardner GJ, Costantino PD, Chi DS, Bogatch K, Chapman PB, Wolchok JD, Schwartz GK and Carvajal RD: Treatment of locally recurrent mucosal melanoma with topical imiquimod. J Clinical Oncol 29: e809811, 2011.

33 Prescott LS, Papadopoulos NE, Euscher ED, Watkins JL and Schmeler KM: Topical treatment of recurrent vaginal melanoma in situ with imiquimod: A case report. Gynecol Oncol Case Rep 2: 92-93, 2012
Received October 24, 2016

Revised October 26, 2016

Accepted November 12, 2016 